\title{
INFLUENCE OF PARAMETERS OF CORE BINGHAM MATERIAL ON CRITICAL BEHAVIOUR OF THREE-LAYERED ANNULAR PLATE
}

\author{
DOROTA PAWLUS
}

Faculty of Mechanical Engineering and Computer Science, University of Bielsko-Biała, Poland, e-mail: doro@ath.bielsko.pl

\begin{abstract}
The paper presents the dynamic response of annular three-layered plate subjected to loads variable in time. The plate is loaded in the plane of outer layers. The plate core has the electrorheological properties expressed by the Bingham body model. The dynamic stability loss of plate with elastic core is determined by the critical state parameters, particularly by the critical stresses. Numerous numerical observations show the influence of the values of viscosity constant and critical shear stresses, being the Bingham body parameters, on the supercritical viscous fluid plate behaviour. The problem has been solved analytically and numerically using the orthogonalization method and finite difference method. The solution includes both axisymmetric and asymmetric plate dynamic modes.
\end{abstract}

Key words: annular sandwich plate, electrorheological model, dynamic stability, finite difference method

\section{INTRODUCTION}

The loss of plate dynamic stability is one of the basic problems in dynamics, which has the theoretical, cognitive and practical, engineering meaning. The evaluation of dynamic instability phenomenon of plate element is strictly connected with its material and geometrical properties. It depends on loading characteristic and accepted criterion of the loss of plate stability. This problem is the complex task both in description of the mathematical formulation and in the process of numerical solution. Complexity of problem and its important practical meaning are the reasons of its scientific current affairs.

The dynamic stability analyses of annular plates with layered structure are subjects of many papers [1]-[5]. The examinations of dynamic stability of such peculiar element like annular shape of plate and complex three-layered structure with the rheological properties of plate layer do not enable to formulate the general evaluations of analysed phenomenon but rather practical remarks for this plate example. Peculiarity of this issue and possible wide range of applications, for example, in aerospace industry, mechanical and nuclear engineering, became the reason of the investigative interest in this work.

In papers [6], [7] the problem of the response of annular plate with symmetrical, cross-sectional structure composed of three layers with the elastic or vis- coelastic core has been presented in detail. The way of analytical and numerical solution based on approximation methods (orthogonalization and finite difference) has been presented. Additionally, in works [6], [8], [9] many numerical analyses using finite element method in ABAQUS system have been presented. It should be noted that presented analytical and numerical solution is general and enables the evaluation of the plate critical state for both axisymmetrical and asymmetrical buckling modes.

Results presented in works [6], [9], [10] for plate structure with viscoelastic core modelled with threeparameter standard model show rather small influence of viscous properties of core material on the plate critical parameters. Meaningful is a very short time passing to the moment of the loss of plate stability and rather high value of viscosity constant of standard core material. The observations showed the changes in dynamic plate behaviour for lower values of core viscosity constant for which its properties change from solid body to viscous fluid.

The known, natural phenomenon of such change is the electrorheological effect, also called the Winslow effect [11]. This rheological behaviour is controlled by the electrical field strength. It concerns some dielectric materials [12]. Approximation of this phenomenon by the Bingham plastic model assumed in description of the core material enables to conduct new evaluation process of plate dynamic behaviour. The dynamic sensitivity of plate structure to different values of Bingham 
model parameters and the analysis of critical and supercritical behaviour could be investigated. Observations and the obtained results indicate that controlling of plate dynamic work is possible. It has a practical importance. The investigations of dynamic behaviours of sandwich annular plates with electrorheological fluid core are presented in works by Yeh [13], [14]. The analyses of free vibrations of isotropic plates [13] and dynamic stability of orthotropic plates [14] concern the full-coverage annular plate. The results show the possibility of active dynamic control.

To the author's knowledge, the current work contains new approach to the dynamic problem of plate loaded in the plane of facings with forces varying in time and plate structure with changeable core material properties from elastic solid body to viscous fluid. The final results obtained in exemplary analyses of the plates show the effectiveness of solution to the problem and the active response of plate with core made of material of various properties.

\section{PROBLEM FORMULATION}

Subject of the analysis is the three-layered annular plate. The plate is composed of thin, elastic facings and the core with electrorheological properties. Plate is loaded on facings with the stress uniformly distributed on perimeter increasing in time according to the linear formula:

$$
p=s t,
$$

where:

$p$-compressive stress,

$s$ - rate of loading growth,

$t$ - time.

The plate edges are slidably clamped. The schematics of the plate is shown in Fig. 1.

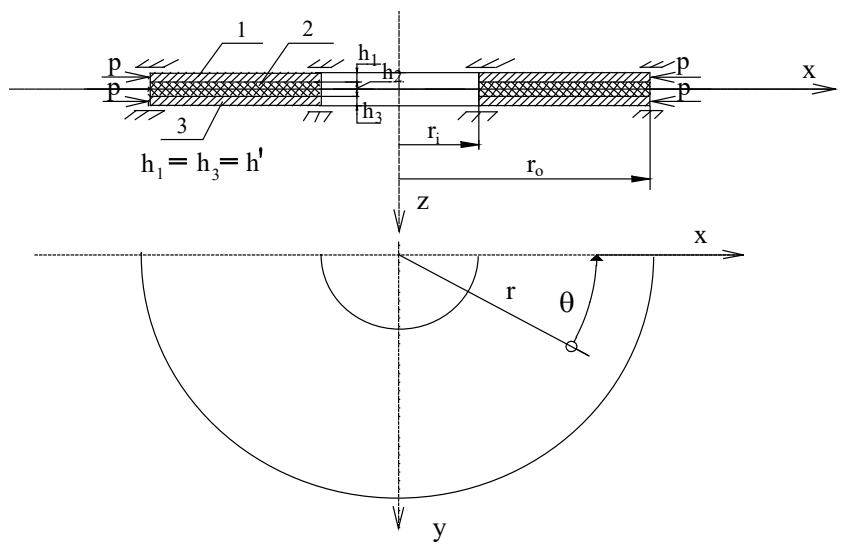

Fig. 1. Schematics of analysed plate
The loading of preliminarily deflected plate acting in facings plane causes the increase in plate dynamic deflections. In the area of strongly increasing deflections the plate loses its dynamic stability. In the evaluation of critical values of deflections, time and load the criterion of the loss of plate dynamic stability presented in work [15] by Volmir was adopted. According to this criterion, the loss of plate stability occurs at the moment of time when the speed of the plate's point of maximum deflection reaches the first maximum value.

The influence of electrorheological properties of plate's core material on dynamic behaviour was examined in the range of critical parameters of plate work. At the moment of time (marked as $t_{c r}$ ), which expresses the loss of plate dynamic stability, the change of core material behaviour occurs from the properties of linear, elastic body to properties of linear, viscous fluidsuspension. This way the damping effect of supercritical dynamic response of loaded plate could be realized. The possibility of some limitation of the range of examined plate work enables the control and monitoring of plate behaviour aiming, for example, for safe application of plate construction element in the area of supercritical and critical loads.

The influence of the parameters of plate material model, particularly viscosity constant of rheological form of core material and the range of shear stress values in core Bingham model on the final results in the area of possible supercritical plate work was subjected to the detailed analysis. The static value of shear stress in the equation of Bingham body (Eq. (6)) has been accepted as equal to values of critical radial and circumferential stresses calculated at the moment of the loss of plate dynamic stability.

In the problem solution and detailed analyses both axisymmetric and circumferentially waved forms of plate buckling have been considered. The classical theory of sandwich plates with the broken line hypothesis [16] has been used. The distribution of stresses to normal and shear loading on plate facings and core has been accepted, respectively. The nonlinear geometric relations expressed by Kármán's equations describe the deformation of facings. The preliminary deflections and additional deflections increasing in loading time are equal for each plate layer.

\section{BASIC EQUATIONS}

The main elements of the solution based on the one presented in work [6], are the following: 
a) formulation of the dynamic equilibrium equations for each plate layer;

b) description of the core deformation in radial and circumferential directions calculating the angles $\beta$ and $\alpha$, respectively [6]:

$$
\begin{gathered}
\beta=\frac{u_{1}-u_{3}}{h_{2}}-\frac{1}{2} \frac{h_{1}+h_{3}}{h_{2}} w_{d, r}+w_{o, r}, \\
\alpha=\frac{v_{1}-v_{3}}{h_{2}}-\frac{1}{2 r} \frac{h_{1}+h_{3}}{h_{2}} w_{d, \theta}+\frac{1}{r} w_{o, \theta},
\end{gathered}
$$

where:

$w_{d}$ - additional plate deflection,

$w_{o}$ - preliminary plate deflection,

$h_{1(3)}, h_{2}$ - facings and core thickness, respectively,

$u_{1(3)}, v_{1(3)}$ - displacements of the points of the mid-

dle plane of facings in the radial and circumferential directions, respectively,

c) application for facings of the linear physical relations of Hook's law and for plate core material of linear elastic and linear viscous flow relations. The physical relations of elastic core material subjected to shearing stresses are expressed by the equations:

$$
\tau_{r z_{2}}=G_{2} \cdot \gamma_{r z_{2}} \quad \tau_{\theta z_{2}}=G_{2} \cdot \gamma_{\theta z_{2}}
$$

where:

$\gamma_{r z_{2}}, \gamma_{\theta z_{2}}$ are the shearing strains of plate core in radial and circumferential direction, respectively, expressed by:

$$
\gamma_{r z_{2}}=u_{2, z}^{(z)}+w_{d, r} \quad \gamma_{\theta z_{2}}=v_{2, z}^{(z)}+\frac{1}{r} w_{d, \theta},
$$

$u_{2}^{(z)}=u_{2}-z \beta+z w_{o, r}, \quad v_{2}^{(z)}=v_{2}-z \alpha+z \frac{1}{r} w_{o, \theta}$ are the radial and circumferential displacements of the points in the $z$ distance between the point and the middle surface of plate core,

$u_{2}, v_{2}$ - displacements of the points of the middle plane of core layer in the radial and circumferential direction, respectively,

$G_{2}-$ Kirchhoff's modulus.

Physical relations of Bingham body core material are expressed by equations [11], [12]:

$$
\tau_{r z_{2}}=\eta \cdot \dot{\gamma}_{r z_{2}}+\tau_{r \max }, \quad \tau_{\theta z_{2}}=\eta \cdot \dot{\gamma}_{\theta z_{2}}+\tau_{\theta \max }
$$

where:

$\eta$ - plastic viscosity constant,

$\dot{\gamma}_{r z(\theta z)_{2}}$ - shear rate in radial and circumferential directions, respectively,

$\tau_{r(\theta) \max }-$ static yield stress in radial and circumferential directions, respectively, d) establishing the sectional forces and moments in facings using the equations of the nonlinear Kármán's plate;

e) formulation of the resultant transverse radial $Q_{r}$ and circumferential $Q_{\theta}$ forces using the core transverse forces $Q_{r 2}, Q_{\theta_{2}}$ expressed by the formulas $Q_{r_{2}}=\tau_{r z_{2}} h_{2}, Q_{\theta_{2}}=\tau_{\theta z_{2}} h_{2}$ in the following form:

$$
\begin{gathered}
Q_{r_{2}}=\widetilde{G}_{2}\left(\delta+H^{\prime} w_{d^{\prime} r}\right)+\widetilde{Q}_{r 2}, \\
Q_{\theta_{2}}=\widetilde{G}_{2}\left(\gamma+H^{\prime} \frac{1}{\mathrm{r}} w_{d^{\prime} \theta}\right)+\widetilde{Q}_{\theta 2}
\end{gathered}
$$

where:

$\delta, \gamma$-differences of radial $\left(u_{3}-u_{1}\right)$ and circumferential $\left(v_{3}-v_{1}\right)$ displacements of the points in middle surfaces of facings, respectively,

$H^{\prime}=h^{\prime}+h_{2}, h^{\prime}=h_{1}=h_{3}$ - equal thickness of facings,

$\widetilde{G}_{2}=G_{2}$ and $\widetilde{Q}_{r 2}=\widetilde{Q}_{\theta 2}=0$ for the elastic core,

$\widetilde{G}_{2}=\eta \frac{\partial}{\partial t}, \frac{\partial}{\partial t}-$ differential operator, $\widetilde{Q}_{r 2}=\tau_{r \max } h_{2}$, $\widetilde{Q}_{\theta_{2}}=\tau_{\theta \max } h_{2}$ for electrorheological core material.

The resultant forces $Q_{r}, Q_{\theta}$ as the sums of the individual layer forces $Q_{r_{1(2,3)}}, Q_{\theta_{1(2,3)}}$ found based upon the equilibrium equations have the following form:

$$
\begin{gathered}
Q_{r}=-k_{1} w_{d^{\prime} r r r}-\frac{k_{1}}{r} w_{d^{\prime} r r}+\frac{k_{1}}{r^{2}} w_{d^{\prime} r}-\frac{k_{2}}{r^{2}} w_{d^{\prime} r \theta \theta} \\
+\frac{k_{1}+k_{2}}{r^{3}} w_{d^{\prime} \theta \theta}+\widetilde{G}_{2}\left(\delta+H^{\prime} w_{d^{\prime} r}\right) \frac{H^{\prime}}{h_{2}}+\frac{\widetilde{Q}_{r^{2}}}{h_{2}} H^{\prime}, \\
Q_{\theta}=-\frac{k_{1}}{r^{3}} w_{d^{\prime} \theta \theta \theta}-\frac{k_{1}}{r^{2}} w_{d^{\prime} \theta r}-\frac{k_{2}}{r} w_{d^{\prime} \theta r r} \\
+\widetilde{G}_{2}\left(\gamma+H^{\prime} \frac{1}{r} w_{d^{\prime} \theta}\right) \frac{H^{\prime}}{h_{2}}+\frac{\widetilde{Q}_{\theta 2}}{h_{2}} H^{\prime},
\end{gathered}
$$

where:

$$
\begin{aligned}
& k_{1}=2 D, k_{2}=4 D_{r \theta}+v k_{1} \\
& D=\frac{E h^{\prime^{3}}}{12\left(1-v^{2}\right)}, D_{r \theta}=\frac{G h^{\prime 3}}{12}-\text { flexural rigidity of }
\end{aligned}
$$
the outer layers,

$E, G, v-$ the equal values of Young's and Kirchhoff's moduli and Poisson's ratio of the facings material, respectively,

f) formulation of the resultant membrane forces expressed by the introduced stress function $\Phi$;

g) determination of the initial loading and boundary conditions and connected with the slidably clamped both inner and outer plate edges: 


$$
\begin{gathered}
\left.w\right|_{t=0}=w_{0}, \quad w_{, t \mid t=0}=0, \quad w_{\left.d\right|_{t=0}}=0,\left.\quad w_{d, t}\right|_{t=0}=0, \\
\left.\sigma_{r}\right|_{r=r_{i}\left(r_{o}\right)}=-p(t) d_{1(2)},\left.\quad \sigma_{r, t}\right|_{r=r_{i}\left(r_{o}\right)}=-(p(t))_{, t} d_{1(2)}, \\
\left.\tau_{r \theta}\right|_{r=r_{i}\left(r_{o}\right)}=0, \\
\left.\right|_{r=r_{i}\left(r_{o}\right)}=0,\left.\quad w_{, r}\right|_{r=r_{i}\left(r_{o}\right)}=0, \quad \delta=\left.\gamma\right|_{r=r_{i}\left(r_{o}\right)}=0, \\
\left.\delta_{, r}\right|_{r=r_{i}\left(r_{o}\right)}=0,
\end{gathered}
$$

where:

$w$ - total deflection,

$\sigma_{r}$ - radial stress, $\tau_{r \theta}-$ shear stress,

$d_{1}, d_{2}$ - quantities, equalled to 0 or 1 , determining the loading of the inner or/and outer plate perimeter,

h) determination of the basic differential equation expressed the deflections of the analysed sandwich plate with electrorheological core material:

$$
\begin{aligned}
& k_{1} w_{d, r r r r}+\frac{2 k_{1}}{r} w_{d, r r r}-\frac{k_{1}}{r^{2}} w_{d, r r}+\frac{k_{1}}{r^{3}} w_{d, r}+\frac{k_{1}}{r^{4}} w_{d, \theta \theta \theta \theta} \\
& +\frac{2\left(k_{1}+k_{2}\right)}{r^{4}} w_{d, \theta \theta}+\frac{2 k_{2}}{r^{2}} w_{d, r r \theta \theta}-\frac{2 k_{2}}{r^{3}} w_{d, r \theta \theta}-\widetilde{G}_{2} \frac{H^{\prime}}{h_{2}} \frac{1}{r} \\
& \cdot\left(\gamma_{, \theta}+\delta+r \delta_{, r}+H^{\prime} \frac{1}{r} w_{d, \theta \theta}+H^{\prime} w_{d, r}+H^{\prime} r w_{d, r r}\right) \\
& -\frac{H^{\prime}}{h_{2}} \frac{1}{r}\left(r \widetilde{Q}_{r 2^{\prime} r}+\widetilde{Q}_{r 2}+\widetilde{Q}_{\theta 2^{\prime} \theta}\right) \\
& =\frac{2 h^{\prime}}{r}\left(\frac{2}{r^{2}} \Phi_{, \theta} w_{, r \theta}-\frac{2}{r} \Phi_{, r \theta} w_{, r \theta}+\frac{2}{r^{2}} w_{, \theta} \Phi_{, r \theta}-\frac{2}{r^{3}} w_{, \theta} \Phi_{, \theta}\right. \\
& \left.+w_{, r} \Phi_{, r r}+\Phi_{, r} w_{, r r}+\frac{1}{r} \Phi_{, \theta \theta} w_{, r r}+\frac{1}{r} \Phi_{, r r} w_{, \theta \theta}\right)-M w_{d, t t},
\end{aligned}
$$

where:

$\Phi$ - stress function,

$M$ - expression: $M=2 h^{\prime} \mu+h_{2} \mu_{2}$,

$\mu, \mu_{2}-$ facing and core mass density, respectively.

\section{PROBLEM SOLUTION}

The quantities $\delta$ and $\gamma$, unknown in equation (11), have been obtained determining the differences in the radial and circumferential displacements $u_{1}, u_{3}$ and $v_{1}$, $v_{3}$ of points from the middle surface of the plate facings (Eq. (8)) establishing the equilibrium equations for forces acting on the undeformed outer plate layers in the $u$ and $v$ direction. Using the equations of membrane $N_{r_{i}}, N_{\theta_{i}}, T_{r \theta_{i}}$ sectional forces and expressions of sums of stresses $\tau_{r_{1}}, \tau_{r_{3}}$ and $\tau_{\theta_{1}}, \tau_{\theta_{3}}$ based on the equilib- rium equations: $\tau_{r_{1}}+\tau_{r_{3}}=\frac{2}{h_{2}} Q_{r_{2}}, \quad \tau_{\theta_{1}}+\tau_{\theta_{3}}=\frac{2}{h_{2}} Q_{\theta_{2}}$ after some transformations, the following differential equations have been found:

$$
\begin{aligned}
& \frac{2 r}{h_{2}} \widetilde{G}_{2} H^{\prime} w_{d^{\prime} r}=\frac{E h^{\prime}}{1-v^{2}}\left(r \delta_{r_{r}}+\delta_{r_{r}}-\frac{1}{r} \delta+v \gamma_{{ }^{\prime} \theta}-\frac{1}{r} \gamma^{\prime} \theta\right) \\
& +G H^{\prime} \frac{1}{r}\left(\delta_{\cdot \theta \theta}+r \gamma_{{ }^{\prime} r \theta}-\gamma_{\cdot \theta}\right)-\frac{2 r}{h_{2}} \widetilde{G}_{2} \delta-\frac{2 r}{h_{2}} Q_{r 2}, \\
& \frac{2}{h_{2}} \widetilde{G}_{2} H^{\prime} w_{d^{\prime} \theta}=\frac{E h^{\prime}}{1-v^{2}}\left(\frac{1}{r} \delta_{r^{\prime}}+v \delta_{r^{\prime} \theta}+\frac{1}{r} \gamma^{\prime} \theta \theta\right) \\
& -\frac{2 r}{h_{2}} \widetilde{G}_{2} \gamma-\frac{2 r}{h_{2}} \widetilde{Q}_{\theta 2}+G h^{\prime} \frac{1}{r}\left(\delta_{r_{\theta}}+r \delta_{r_{r} \theta}+r^{2} \gamma_{r_{r r}}-\gamma\right) .
\end{aligned}
$$

In the solution the following dimensionless quantities and the expressions have been accepted [7], [17]:

$$
\begin{gathered}
\zeta=\frac{w}{h} \zeta_{1}=\frac{w_{d}}{h} \zeta_{0}=\frac{w_{0}}{h} F=\frac{\Phi}{E h^{2}} \\
\rho=\frac{r}{r_{0}} \bar{\delta}=\frac{\delta}{h} \bar{\gamma}=\frac{\gamma}{h} t^{*}=t \cdot K 7 \quad K 7=\frac{s}{P_{c r}} \\
\zeta_{1}(\rho, \theta, t)=X_{1}(\rho, t) \cos (m \theta) \\
\zeta_{0}(\rho, \theta)=X_{a}(\rho)+X_{b}(\rho) \cos (m \theta), \\
\zeta=\zeta_{1}+\zeta_{0} \\
F(\rho, \theta, t)=F_{a}(\rho, t)+F_{b}(\rho, t) \cos (m \theta) \\
+F_{c}(\rho, t) \cos (2 m \theta) \\
\bar{\delta}(\rho, \theta, t)=\bar{\delta}(\rho, t) \cos (m \theta) \\
\bar{\gamma}(\rho, \theta, t)=\bar{\gamma}(\rho, t) \sin (m \theta) \\
\tau_{r \max }(\rho, \theta)=\tau_{r \max }(\rho) \cos (m \theta) \\
\tau_{\theta \max }(\rho, \theta)=\tau_{\theta \max }(\rho) \sin (m \theta)
\end{gathered}
$$

where:

$r_{0}$ - outer radius,

$m$ - number of circumferential waves corresponding to the form of plate buckling,

$h=h_{1}+h_{2}+h_{3}-$ total thickness of plate,

$p_{c r}-$ critical, static stress,

The assumed form of predeflection $\zeta_{0}-$ Eqs. (14) - is expressed by the following formulas presented by Trombski and Wojciech [17]:

$$
X_{a}(\rho)=\xi_{1} \eta(\rho), \quad X_{b}(\rho)=\xi_{2} \eta(\rho)
$$

where:

$\xi_{1}, \xi_{2}-$ calibrating numbers, 
$\eta(\rho)=\rho^{4}+A_{1} \rho^{2}+A_{2} \rho^{2} \ln \rho+A_{3} \ln \rho+A_{4}, A_{i}$ - quantities fulfilling the conditions of clamped edges by the function $\eta(\rho), i=1,2,3,4$.

Using the orthogonalization method to eliminate the angular variable $\theta$, the Eq. (11) has been replaced by the approximate one obtained from the following condition:

$$
\int_{0}^{2 \pi} \psi \cos (m \theta) d \theta=0,
$$

where $\psi$-is the difference of the left and right side of the transformed Eq. (11).

Using the physical relations, relations of sectional forces and moments in facings with stresses and equations of resultant membrane forces expressed by the introduced stress function after the elimination of the quantities described by the sums of the radial $\left(u_{1}+u_{3}\right)$ and circumferential $\left(v_{1}+v_{3}\right)$ facing displacements the following equation has been obtained:

$$
\begin{gathered}
\Phi_{, r r r r}+\frac{2}{r} \Phi_{, r r r}-\frac{1}{r^{2}} \Phi_{, r r}+\frac{1}{r^{3}} \Phi_{, r}+\frac{1}{r^{4}} \Phi_{, \theta \theta \theta \theta} \\
+\frac{4}{r^{4}} \Phi_{, \theta \theta}-\frac{2}{r^{3}} \Phi_{, r \theta \theta}+\frac{2}{r^{2}} \Phi_{, r r \theta \theta} \\
=E\left[\frac{1}{r} w_{o, r r}\left(w_{o, r}+\frac{1}{r} w_{o, \theta \theta}\right)+-\frac{1}{r^{2}}\left(\frac{1}{r} w_{o, \theta}-w_{o, r \theta}\right)^{2}\right. \\
\left.-\frac{1}{r} w_{, r r}\left(w_{, r}+\frac{1}{r} w_{, \theta \theta}\right)+\frac{1}{r^{2}}\left(\frac{1}{r} w_{, \theta}-w_{, r \theta}\right)^{2}\right] .
\end{gathered}
$$

After the insertion of the Equations (14), (15) describing the nondimensional expressions of functions $w, w_{0}, \Phi$ and after the comparison of the expressions by the same trigonometric functions, the Equation (18) has been presented in the form of the three equations.

In the solution, the finite difference method has been used for approximation of the derivatives with respect to $\rho$ by the central differences in the discrete points. The form of the system of differential equations expressing the deflections of three-layered, annular plate with electrorheological core is as follows:

$$
\begin{aligned}
\mathbf{P U}+\mathbf{Q}+\mathbf{P}_{L} \dot{\mathbf{U}}+\mathbf{Q}_{L}+Q_{E}=K_{E} \ddot{\mathbf{U}}, \\
\mathbf{M}_{Y} \mathbf{Y}=\mathbf{Q}_{Y}, \\
\mathbf{M}_{Y} \dot{\mathbf{Y}}=\dot{\mathbf{Q}}_{Y}, \\
\mathbf{M}_{V} \mathbf{V}=\mathbf{Q}_{V},
\end{aligned}
$$

$$
\begin{gathered}
\mathbf{M}_{V} \dot{\mathbf{V}}=\dot{\mathbf{Q}}_{V}, \\
\mathbf{M}_{Z} \mathbf{Z}=\mathbf{Q}_{Z}, \\
\mathbf{M}_{Z} \dot{\mathbf{Z}}=\dot{\mathbf{Q}}_{Z}, \\
\mathbf{M}_{D L} \dot{\mathbf{D}}=\mathbf{M}_{D} \mathbf{D}+\mathbf{M}_{U} \mathbf{U}+\mathbf{M}_{U L} \dot{\mathbf{U}} \\
+\mathbf{M}_{G} \mathbf{G}+\mathbf{M}_{G L} \dot{\mathbf{G}}+\mathbf{E R}, \\
\mathbf{M}_{G G L} \dot{\mathbf{G}}=\mathbf{M}_{G G} \mathbf{G}+\mathbf{M}_{G U} \mathbf{U}+\mathbf{M}_{G U L} \dot{\mathbf{U}} \\
+\mathbf{M}_{G D} \mathbf{D}+\mathbf{M}_{G D L} \dot{\mathbf{D}}+\mathbf{E} \Theta,
\end{gathered}
$$

where:

$\mathbf{Q}_{E}-$ vector of elements expressed as: $\frac{H^{\prime}}{h_{2} h} W K 5\left(\frac{m}{\rho} \tau_{\theta \max _{i}}+\frac{1}{\rho} \tau_{r \max _{i}}+\tau_{r \max _{i^{\prime} \rho}}\right)$,

$K_{E}=\mathrm{K} 7^{2} \mathrm{WK} 5 \mathrm{WK} 8^{\prime}$,

WK5, WK8' - expressions: WK5 $=\frac{h^{\prime}}{h}, \quad$ WK $8^{\prime}$ $=r_{0} h_{2} M$,

$\mathbf{U}, \mathbf{Y}, \mathbf{V}, \mathbf{Z}, \dot{\mathbf{U}}, \ddot{\mathbf{U}}, \ddot{\mathbf{U}}, \dot{\mathbf{Y}}, \dot{\mathbf{V}}, \dot{\mathbf{Z}}$ - vectors and their derivatives with respect to time $t$ of plate additional deflections and components $F_{a}, F_{b}, F_{c}$ of the stress function $F_{a_{\rho}}=y, F_{b}=v, F_{c}=z$, respectively,

$\mathbf{P}, \mathbf{P}_{L}-$ matrices with elements and its derivative with respect to time $t$, which are composed of geometric and material plate parameters, the quantity $b$ (length of the interval in the finite difference), $m$ (the number of buckling waves), dimensionless radius $\rho$, respectively,

$\mathbf{Q}, \mathbf{Q}_{L}$ - vectors of expressions and its derivative with respect to time $t$, which are composed of the initial and additional deflections, geometric and material parameters, components of the stress function, radius $\rho$, quantity $b$, coefficients $\delta, \gamma$ and number $m$, respectively,

$\mathbf{M}_{Y}$ - matrix of elements composed of the radius $\rho$ and quantity $b$, respectively,

$\mathbf{M}_{V}, \mathbf{M}_{Z}$ - matrices of elements composed of the radius $\rho$, quantity $b$ and number $m$,

$\mathbf{Q}_{Y}, \mathbf{Q}_{V}, \mathbf{Q}_{Z}, \dot{\mathbf{Q}}_{Y}, \dot{\mathbf{Q}}_{V}, \dot{\mathbf{Q}}_{Z}$ - vectors of expressions and their derivatives with respect to time $t$, which are composed of the initial and additional deflections, radius $\rho$, quantity $b$ and number $m$, respectively,

$\mathbf{M}_{D}, \mathbf{M}_{G}, \mathbf{M}_{G G}, \mathbf{M}_{G D}, \dot{\mathbf{M}}_{D L}, \dot{\mathbf{M}}_{G L}, \dot{\mathbf{M}}_{G G L}, \dot{\mathbf{M}}_{G D L}$ - matrices of elements and their derivatives with respect to time, which are composed of geometric and material parameters, quantity $b$ and number $m t$, respectively, 
$\mathbf{M}_{U}, \dot{\mathbf{M}}_{U}$ - matrices of elements and its derivative with respect to time $t$, which are composed of material parameters and quantity $b$, respectively,

$\mathbf{M}_{G U}, \dot{\mathbf{M}}_{G U L}$ - matrices of elements and its derivative with respect to time $t$, which are composed of geometric parameters, material parameters and number $m$, respectively,

$\mathbf{D}, \mathbf{G}, \dot{\mathbf{D}}, \dot{\mathbf{G}}$ - vectors of expressions and their derivatives with respect to time $t$, which are composed of coefficients $\delta$ and $\gamma$, respectively,

$E R, E \Theta$ - vectors of elements expressed as: $E R=\frac{r_{o}}{h_{2} h H^{\prime}} \tau_{r \max _{i}}, E \Theta=\frac{2 r_{o} \rho}{h_{2}{ }^{2} h} \tau_{\theta \max _{i}}$, respectively.

Eliminating the Eqs. (21), (23), (25) and the expressions connected with the differential operator $\partial / \partial t$ applied in physical relations of electrorheological material of plate core from the system of Eqs. (19)-(27), the system of equations for plate with elastic core is obtained:

$$
\begin{gathered}
\mathbf{P} \mathbf{U}+\mathbf{Q}=K \ddot{\mathbf{U}}, \\
\mathbf{M}_{Y} \mathbf{Y}=\mathbf{Q}_{Y}, \\
\mathbf{M}_{V} \mathbf{V}=\mathbf{Q}_{V}, \\
\mathbf{M}_{Z} \mathbf{Z}=\mathbf{Q}_{Z}, \\
\mathbf{M}_{D} \mathbf{D}=\mathbf{M}_{U} \mathbf{U}+\mathbf{M}_{G} \mathbf{G}, \\
\mathbf{M}_{G G} \mathbf{G}=\mathbf{M}_{G U} \mathbf{U}+\mathbf{M}_{G D} \mathbf{D},
\end{gathered}
$$

where:

$$
\begin{aligned}
& K=\mathrm{K} 7^{2} \mathrm{WK} 5 \mathrm{WK} 8, \\
& \text { WK8 - expression: WK8 }=\mathrm{r}_{\mathrm{o}} M \frac{h_{2}}{G_{2}} .
\end{aligned}
$$

The system of Equations (19)-(27) and (28)-(33) was solved using Runge-Kutta's integration method for the initial state of plate. Critical static stress $p_{c r}$ has been calculated solving the eigenproblem for the problem of the disk state neglecting the inertial components and nonlinear expressions [6]-[8].

\section{NUMERICAL EXAMPLES}

The plate cases loaded on inner or outer edges have been subjected to numerical investigations. The linear, quickly increasing load which acts on plate edge is expressed by the Eq. (1). The rate of loading growth $\mathrm{s}$ is equal to $s \approx 4346 \mathrm{MPa} / \mathrm{s}$ and $s \approx$ $932 \mathrm{MPa} / \mathrm{s}$ for plates loaded on inner or outer pe- rimeter, respectively. The axisymmetrical $m=0$ form of plate loss of stability and circumferentially waved mode $m=7$ have been considered. The observations have been conducted for plates with facing thickness $h^{\prime}$ equal to: $h^{\prime}=1 \mathrm{~mm}$, core thickness equal to $h_{2}=2 \mathrm{~mm}$, inner radius $r_{i}=0.2 \mathrm{~m}$, outer radius $r_{0}=$ $0.5 \mathrm{~m}$. Facings are made of steel of the following parameters: Young's modulus $E=2.1 \cdot 10^{5} \mathrm{MPa}$, Poisson's ratio $v=0.3$. Generally, core material is expressed by two accepted values of Kirchhoff's modulus $G_{2}$ equal to $G_{2}=0.5 \mathrm{MPa}$ and $G_{2}=2.5 \mathrm{MPa}$ with adopted value of mass density $\mu=64 \mathrm{~kg} / \mathrm{m}^{3}$. The influence of values of viscosity constants $\eta$ of rheological core material on final results was analysed in wide range of values from $\eta=1.7 \mathrm{~Pa} \cdot \mathrm{s}$ to $\eta=1700 \mathrm{~Pa} \cdot \mathrm{s}$ concentrating the numerical examinations for values: from $\eta=10 \mathrm{~Pa} \cdot \mathrm{s}$ to $\eta=17 \mathrm{~Pa} \cdot \mathrm{s}$. For example, plate core material with parameters $G_{2}=2.5 \mathrm{MPa}$ in elastic field behaviour and $\eta=17 \mathrm{~Pa} \cdot \mathrm{s}$ for rheological fluid core properties, the influence of shear stresses values fluctuation (expressed by the formula: $\tau_{c r}$.number) on the supercritical run of curves $\zeta_{1 \max }=f(t)$ was evaluated in detail.

Figure 2 presents the time histories of deflections $\zeta_{1 \max }=f\left(t^{*}\right)$ of plates with elastic core expressed by the Kirchhoff's modulus: $G_{2}=0.5,2.5,5$ and $10 \mathrm{MPa}$ and plates with electrorheological core properties described by modulus $G_{2}=2.5 \mathrm{MPa}$ and values of viscosity constant $\eta=10,1700 \mathrm{~Pa} \cdot \mathrm{s}$. Dimensionless time $t^{*}$ expressed by the respective equation (13) and assumed rate of loading growth $s$ is equal to $t^{*}=t \cdot 20$. Plate is loaded on inner edge. The buckling mode is axisymmetric $m=0$. The curves show the behaviour of plate with elastic core, quick deflections increase in plate critical state, the moment of the loss of plate dynamic stability assigned according to accepted criterion (see, point 2 of this work) - marked point on curve $\zeta_{1 \max }=f\left(t^{*}\right)$ for plate core with value of modulus $G_{2}=2.5 \mathrm{MPa}$ and supercritical plate vibrations initiated by increasing load. Along with the increase in value of modulus $G_{2}$, the plate deflections decrease and the plate becomes stiffer. For plate case with value of core modulus $G_{2}$ equal to $G_{2}=2.5 \mathrm{MPa}$ the defendence of deflections as a finction of time $\zeta_{1 \max }=f\left(t^{*}\right)$ for plate with electrorheological properties of core material (described as el-rh core) have been shown.

Figure 3 shows the influence of values in the range of $\eta=10 \div 17 \mathrm{~Pa} \cdot \mathrm{s}$ of viscosity constant $\eta$ on the run of critical and supercritical deflections. In detail, the results for these plates examples are presented in Fig. 3b in narrow region of plate work expressed by nondimensional time $t^{*}$ equal to: $t^{*}=0.2 \div 0.5$. The increase 


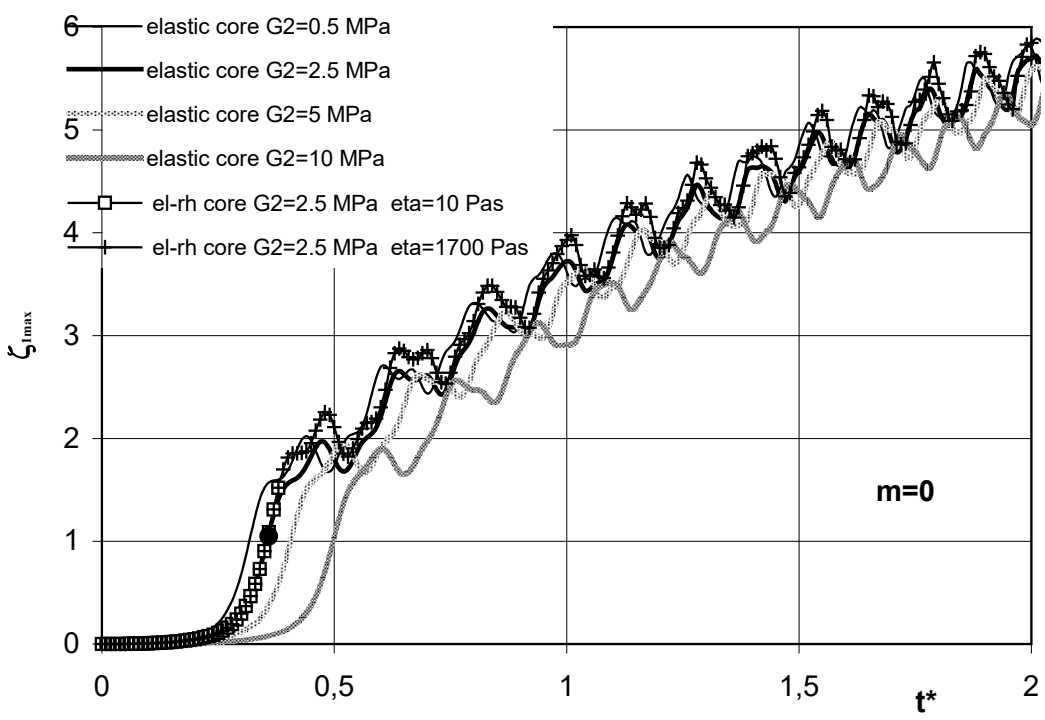

Fig. 2. Dependence of deflections on time for plates $m=0$ with elastic and electrorheological core loaded on inner edge

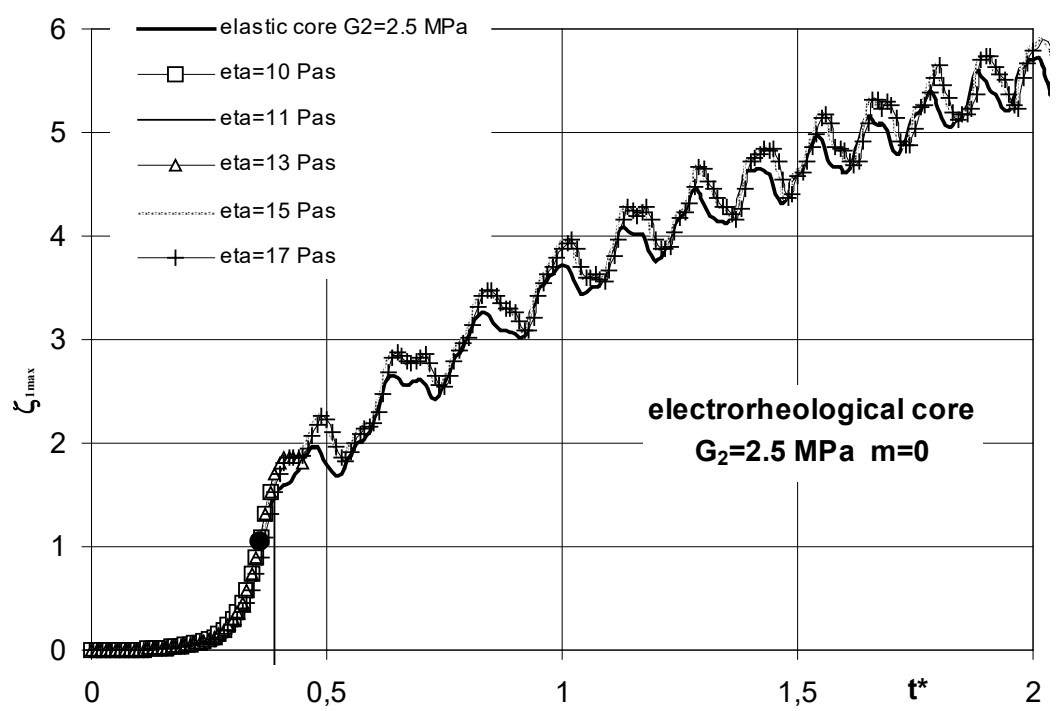

a)

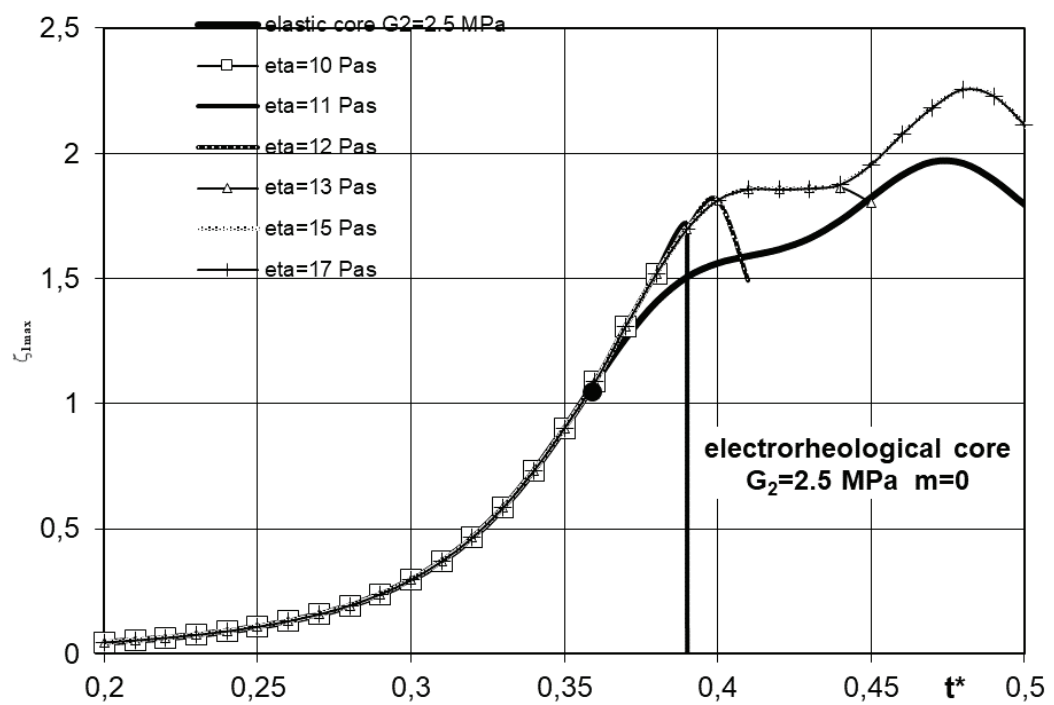

b)

Fig. 3. Dependence of deflections on time for plates $G_{2}=2.5 \mathrm{MPa}, m=0$ loaded on inner edge with electrorheological core for values $\eta=10 \div 17 \mathrm{~Pa} \cdot \mathrm{s}: \mathrm{a}$ ) in full examined range of plate work, b) in the range of plate work expressed by time $t^{*}=0.2 \div 0.5$ 
in values of viscosity constant $\eta$ increases the structure stiffness. The plate behaviour in the range of supercritical vibrations approaches the plates with elastic core. However, supercritical deflections of plate with elastic core are lower than plate with electrorheological core. It is obvious because the plate with electrorheological core properties is flabbier. For a lower values of viscosity constant in the range of $\eta=10 \div 15 \mathrm{~Pa} \cdot \mathrm{s}$ the possible limitation of plate work in critical area is observed.

The influence of values of stress $\tau_{\max }(6)$, which characterizes the model of Bingham body, on the behaviour of plate with core parameters $G_{2}=2.5 \mathrm{MPa}$, $\eta=17 \mathrm{~Pa} \cdot \mathrm{s}$ is presented in Figs. $4 \mathrm{a}$ and $\mathrm{b}$. Results shown in Fig. $4 \mathrm{~b}$ are focused on critical area of plate work.

In presented analyses the values of stress $\tau_{\max }$ in radial $\tau_{\text {rmax }}$ and circumferential $\tau_{\text {tmax }}$ plate directions calculated as the critical values $\tau_{c r}$ (talcr) at the moment of the loss of plate dynamic stability are presented. The distribution of radial critical stress $\tau_{c r}$ for examined, axisymmetric plate is presented in Fig. 5. The circumferential, critical stresses are equal to zero.

The changes of values of stresses $\tau_{r(\theta) \max }$ expressed by the product ( $\tau_{c r}$ number) admittedly influence the run of curves $\zeta_{1 \max }=f\left(t^{*}\right)$ in critical area of plate work. The observations show that possible theoretical increase in values of stresses $\tau_{r(\theta) \max }$ can limit the supercritical plate work. Results show the significant influence of values of Bingham core stresses $\tau_{r(\theta) \max }$ on dynamic plate behaviour. The evaluation of value of this Bingham model parameter with its experimental determination for the structure with electrorheological material is particularly important.

a)
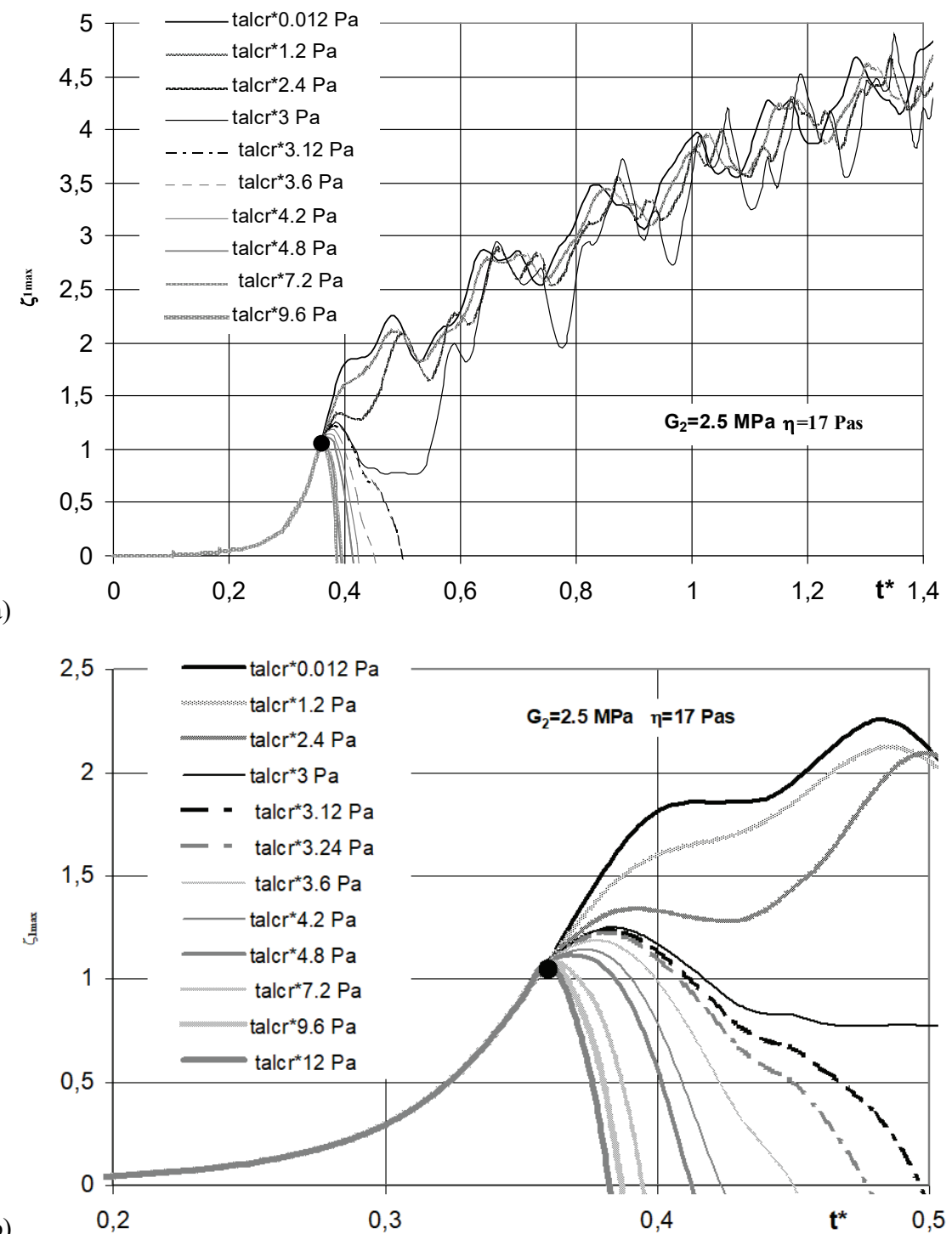

Fig. 4. Dependence of deflections on time for plates with electrorheological core $G_{2}=2.5 \mathrm{MPa}$, $\eta=17 \mathrm{~Pa} \cdot \mathrm{s}, m=0$ loaded on inner edge depending on values of Bingham shear stress:

a) in full examined range of plate work, b) in the range of plate work expressed by time $t^{*}=0.2 \div 0.5$ 


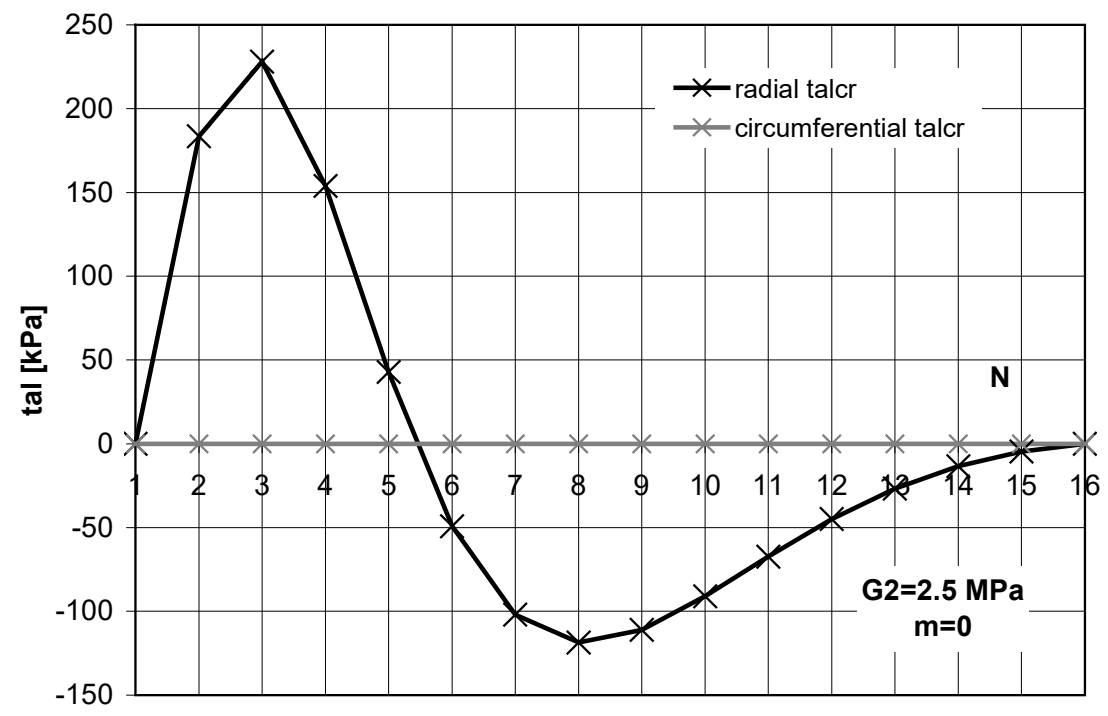

Fig. 5. Distribution of values of critical stresses $\tau_{c r}$ depending on discrete points distribution $N$ for plate $G_{2}=2.5 \mathrm{MPa}, m=0$ loaded on inner edge

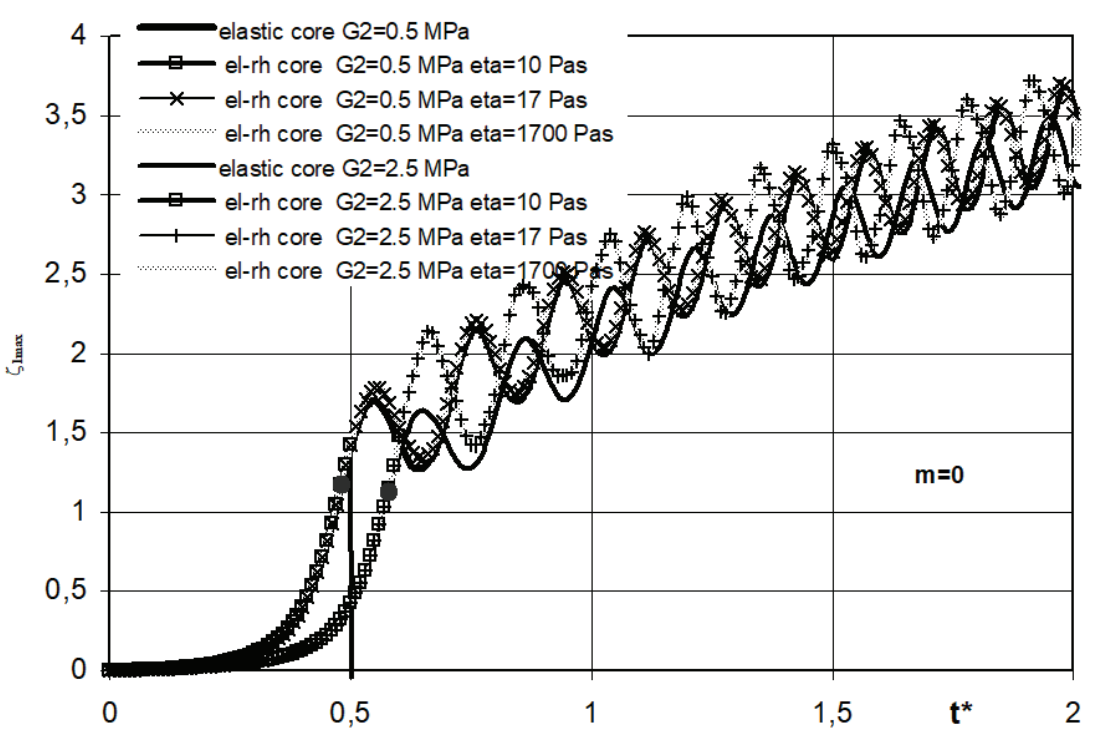

Fig. 6. Dependence of deflections on time for axisymmetric plates $\left(G_{2}=0.5 \mathrm{MPa}, G_{2}=2.5 \mathrm{MPa}\right)$ with elastic and viscous core compressed on outer edge

The run of curves $\zeta_{1 \max }=f\left(t^{*}\right)$ for axisymmetric plate $(m=0)$ compressed on outer edge with elastic and electrorheological properties of core material is presented in Fig. 6. The values of Kirchhoff's modulus of core are $G_{2}=0.5 \mathrm{MPa}$ and $G_{2}=2.5 \mathrm{MPa}$, respectively. In each of analysed plate examples the vibrations initiated in supercritical region of plate work can be observed. In this area the deflections differences between the plate with elastic core and plate with viscous properties are stronger marked for stiffer plate structure with greater value of modulus $G_{2}=2.5 \mathrm{MPa}$. According to prediction of the run of supercritical vibrations of plates with viscous structure is located in fixed band of deflections values. Practically, their level does not depend on the values of Kirchhoff's modulus $G_{2}$ of elastic core material. For viscosity constant $\eta$ equal to $\eta=10 \mathrm{~Pa} \cdot \mathrm{s}$ the region of plate work is limited to the critical load range.

The examples of plates compressed on outer edge, whose buckling is in the form of $m=7$ circumferential waves, are shown in Figs. 7 and 8 for values of Kirchhoff's modulus equal to $G_{2}=2.5 \mathrm{MPa}$ and $G_{2}=0.5 \mathrm{MPa}$, respectively. The plate with value of 
Kirchhoff's modulus equal to $G_{2}=2.5 \mathrm{MPa}$ loses its dynamic stability with the minimal (important in buckling problems) value of critical dynamic load $p_{c r \text { dyn }}$ for the number of circumferential waves $m=7$. The minimal value of $p_{c r \text { dyn }}$ for plate with value $G_{2}=0.5 \mathrm{MPa}$ corresponds to buckling mode $m=6$. Results presented in Fig. 8 are for plate buckling mode $m=7$. The difference of values of critical loads $p_{c r \text { dyn }}$ between value calculated for plate with number $m=6$ and $m=7$ is small. Table 1 presents the values of some critical loads $p_{c r d y n}$ for analysed plates $G_{2}=2.5 \mathrm{MPa}$ and $G_{2}=0.5 \mathrm{MPa}$. Results shown in Figs. 7 and 8 enable the comparison of plates responses. They concern the same form of plate buckling.
Table 1. Critical dynamic loads $p_{c r \text { dyn }}$ corresponding to buckling mode $\mathrm{m}$ of plates with core parameters $G_{2}=2.5 \mathrm{MPa}$ and $G_{2}=0.5 \mathrm{MPa}$

\begin{tabular}{|c|c|c|}
\hline \multirow{2}{*}{$\begin{array}{c}\text { Buckling mode } \\
m\end{array}$} & \multicolumn{2}{|c|}{$\begin{array}{c}\text { Critical dynamic loads } \\
p_{\text {crdyn }}[\mathrm{MPa}]\end{array}$} \\
\cline { 2 - 3 } & $G_{2}=2.5 \mathrm{MPa}$ & $G_{2}=0.5 \mathrm{MPa}$ \\
\hline 0 & 26.97 & 22.40 \\
\hline 3 & 18.77 & 15.51 \\
\hline 5 & 14.95 & 12.06 \\
\hline 6 & 14.30 & $\mathbf{1 1 . 6 0}$ \\
\hline 7 & $\mathbf{1 4 . 1 1}$ & $\mathbf{1 1 . 6 9}$ \\
\hline 8 & 14.77 & 11.97 \\
\hline 9 & 15.23 & 12.81 \\
\hline 10 & 16.72 & 13.46 \\
\hline
\end{tabular}

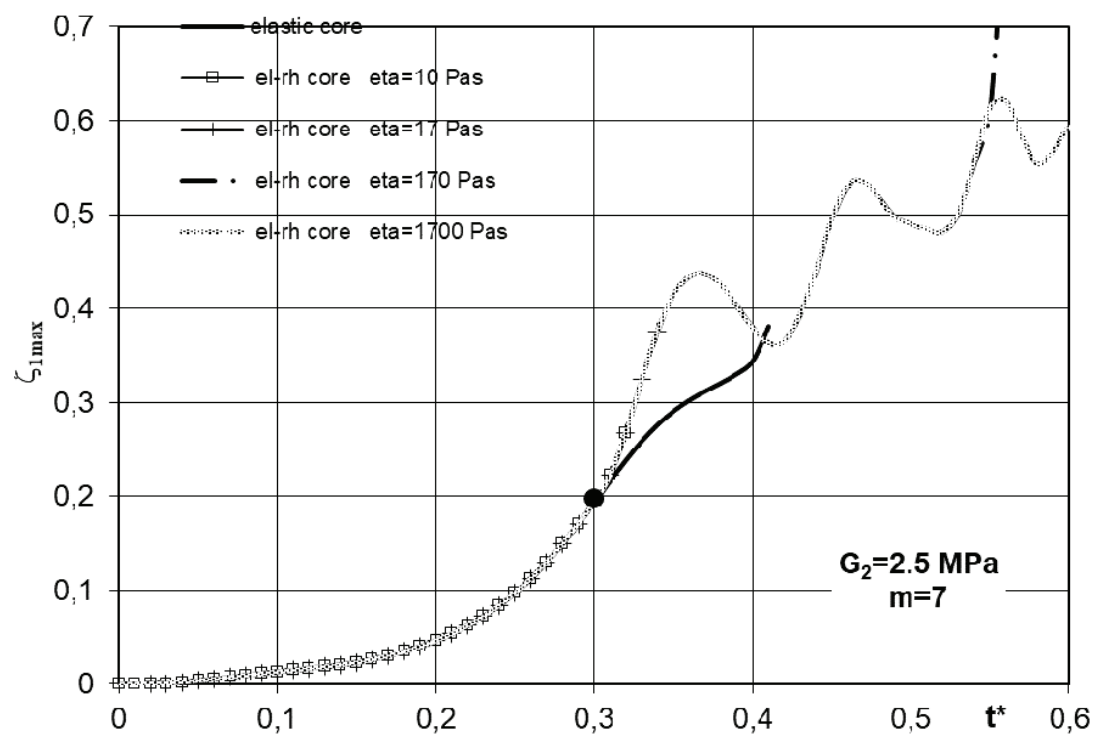

Fig. 7. Dependence of deflections on time for waved plates $G_{2}=2.5 \mathrm{MPa}, m=7$ with elastic and electrorheological core compressed on outer edge

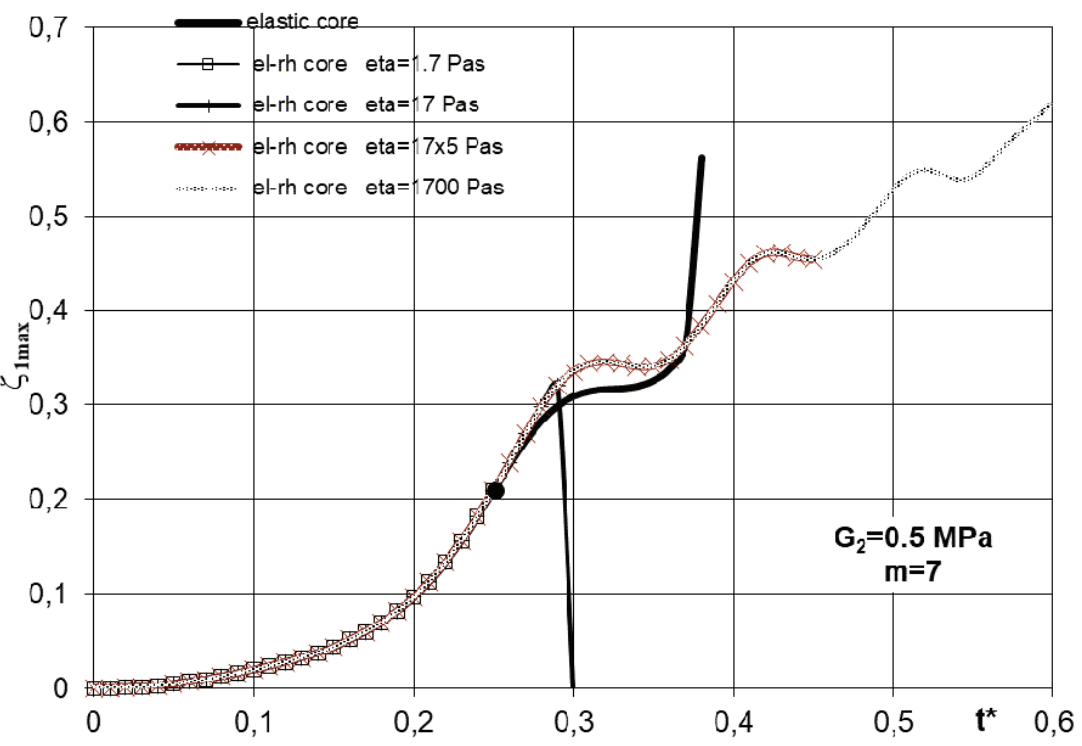

Fig. 8. Dependence of deflections on time for waved plates $G_{2}=0.5 \mathrm{MPa}, m=7$ with elastic and electrorheological core compressed on outer edge 


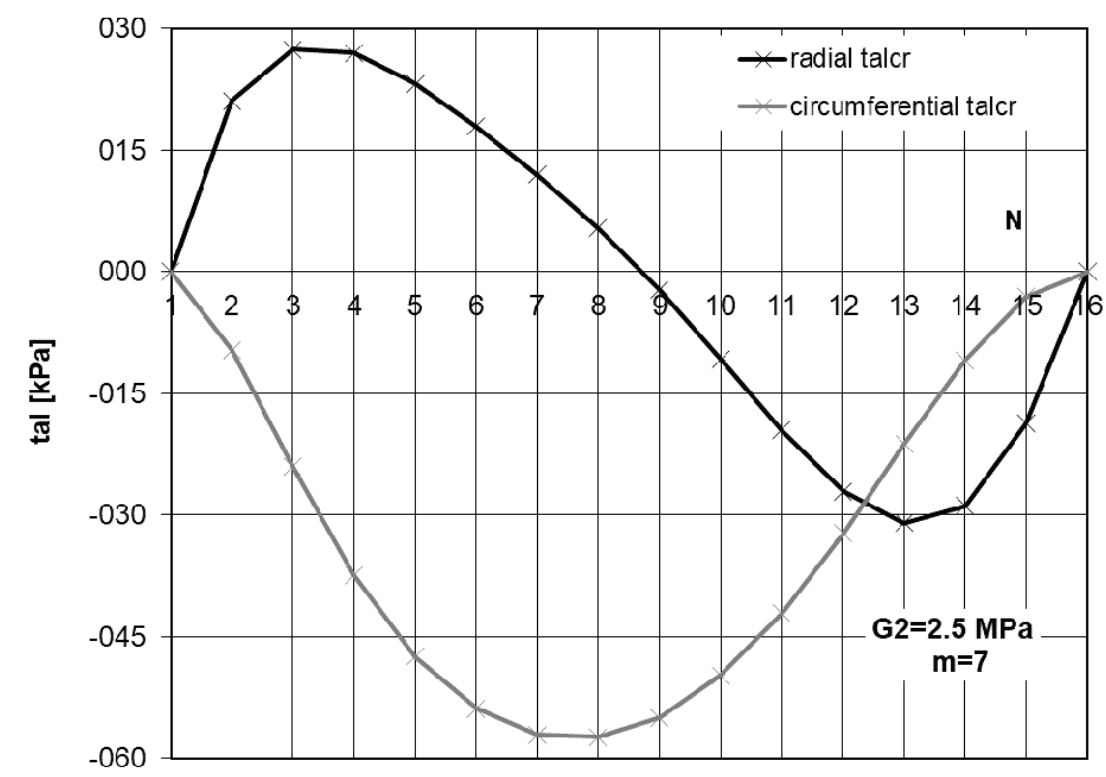

Fig. 9. Distribution of values of critical radial and circumferential shear stresses $\tau_{c r}$ depending on discrete points distribution $N$ for plate $G_{2}=2.5 \mathrm{MPa}, m=7$ compressed on outer edge

The supercritical vibrations shown in Figs. 7 and 8 are observed in common in previous presented plates examples with electrorheological core for greater values of viscosity constant $\eta$ than $\eta=17 \mathrm{~Pa}$.s. The vibration amplitude of plates with lower value of modulus $G_{2}$ is smaller (Figs. 7 and 8). For the smaller value of viscosity constant $\eta$ the run of curves $\zeta_{1 \max }=f\left(t^{*}\right)$ in supercritical region of plate work is limited. Presented analyses were carried out for values of stresses $\tau_{\max }$ of Bingham body core material corresponding to values of critical stresses calculated at the critical time $t_{c r}$. The distribution of values of shear radial $\tau_{\text {rmax }}$ and circumferential $\tau_{\theta \max }$ stresses (Eq. (6)) depending on discrete points distribution $N$ of applied finite difference method for plate model with buckling mode $m=7$ is presented in Fig. 9.

\section{CONCLUSIONS}

A lot of numerical analyses of annular, three-layered plates with electrorheological properties of core material show the dynamic response of plate to used load and various values of parameters, which characterize Bingham model accepted as core material. Electric field changes the properties of electrorheological material from elastic one to characterizing by the viscous flow. The kind of core electrorheological material and the level of electrical field strength in a basic way influence on plate behaviour. The examinations of real element require different, perceptive evaluation of values of material properties. Essential are the experimental tests.

Approach to evaluation of analysed plate behaviour presented in this paper enables the recognition of important sensitivity issue of examined structure in dynamic stability problem. The observations show the strong influence of core material properties on plate dynamic, supercritical behaviour. Results indicate the possibility of controlling of plate work and limitation of the supercritical vibration field. The current results present the huge meaning of values of maximum shear stresses (Eq. (6)), (expressed in work as $\tau_{c r}$ - Fig. 4). For electrorheological material the level of their value depends on the rate of electrical field strength. The changes of values of viscosity constant (Eq. (6)) do not have influence on the run of the curves describing plate deflection in true. This observations confirm the behaviour mechanism of Bingham body material [11].

Undertaken problem is the multiparameter task. The general solution to the problem, presented in this paper, enables the quick, effective evaluation of plate response to different values of parameters describing the plate material geometry and the rate of loading growth. The influence of core thickness and geometry size of the plate on behaviour of structure were presented in work [18].

The problem of dynamic loss of stability of the plate cannot be limited only to axisymmetrical analysis. In many cases the loss of plate stability expressed in the circumferentially waved form. Exemplary results show the behaviour of plates compressed on outer edge with axisymmetric $(m=0)$ and wavy $(m=7)$ 
buckling mode (Figs. 6, 7 and 8, respectively). The rate of deflections of plate with asymmetric buckling mode and the dynamics of its supercritical vibrations change. The analysis including different buckling modes in problem of plane compressed plates is essential.

Presented results and observations could be useful in plate structure design for suitable control of its dynamic behaviour and work field.

\section{REFERENCES}

[1] WANG H.J., CHEN L.W., Vibration and damping analysis of a three-layered composite annular plate with a viscoelastic mid-layer, Composite Structures, 2002, 58, 563-570.

[2] WANG H.J., ChEN L.W., Axisymmetric dynamic stability of sandwich circular plates, Composite Structures, 2003, 59, 99-107.

[3] CHEN Y.R., CHEN L.W., Axisymmetric parametric resonance of polar orthotropic sandwich annular plates, Composite Structures, 2004, 65, 269-277.

[4] CHEN Y.R., CHEN L.W., Vibration and stability of rotating polar orthotropic sandwich annular plates with a viscoelastic core layer, Composite Structures, 2007, 78, 45-57.

[5] CHEN Y.R., CHEN L.W., WANG C.C., Axisymmetric dynamic instability of rotating polar orthotropic sandwich annular plates with a constrained damping layer, Composite Structures, 2006, 73(2), 290-302.

[6] Pawlus D., Dynamic stability of three-layered annular plates with viscoelastic core, Scientific Bulletin of the Technical University of Łódź, Łódź, 2010, 1075, (in Polish).

[7] Pawlus D., Dynamic stability of three-layered annular plates with wavy forms of buckling, Acta Mech., 2011, 216, $123-138$.
[8] Pawlus D., Solution to the problem of axisymmetric and asymmetric dynamic instability of three-layered annular plates, Thin-Walled Structures, 2011, 49, 660-668.

[9] PAwlus D., Critical loads calculations of annular three-layered plates with soft elastic or viscoelastic core, Archives of Civil and Mechanical Engineering, 2011, XI, 4, 993-1009.

[10] Pawlus D., Solution to the Dynamic Stability Problem of ThreeLayered Annular Plate with Viscoelastic Core, Proceedings of the International Association for Shell and Spatial Structures (IASS) Symposium, BEYOND THE LIMITS OF MAN, 23-27 September 2013, Wrocław University of Technology, Poland, J.B. Obrębski and R. Tarczewski (Eds.).

[11] Jordan T.C., SHaw M.T., Electrorheology, Transactions on Electrical Insulation, 1989, Vol. 24, No. 5, 849-878.

[12] YalcinTAS M., DAI H., Magnetorheological and electrorheological materials in adaptive structures and their performance comparison, Smart. Mater. Struc., 1999, 8, 560-573.

[13] YEH J.Y., Vibration control of a sandwich annular plate with an electrorheological fluid core layer, Smart. Mater. Struc., 2007, 16, 837-842.

[14] YeH J.Y., Active dynamic instability control analysis of polar orthotropic sandwich annular plate with electrorheological fluid damping treatment, Journal of Engineering Technology and Education, 2012, Vol. 9, No. 3, 290-299.

[15] Volmir C., Nonlinear dynamic of plates and shells, Science, Moskwa, 1972, (in Russian).

[16] VolmiR C., Stability of deformed system, Science, Moscow, 1967, (in Russian).

[17] TROMBSKi M., Wojciech S., The cylindrically orthotropic annular plate subjected to time-dependent pressure acting in its plane, The Archive of Mechanical Engineering, 1981, Vol. XXVIII, 2, 161-181, (in Polish).

[18] Pawlus D., Dynamic response control of three-layered annular plate due to various parameters of electrorheological core, Archive of Mechanical Engineering, 2016, Vol. LXIII, 1, 73-91. 